\title{
Aspectos morfológicos da cérvice de ovelhas ${ }^{1}$
}

\author{
Diego S. Moura ${ }^{2}$, Tarcísio T. Lourenço², Murillo M. Moscardini², Caroline Scott², \\ Phellipe O. Fonseca ${ }^{2}$ e Fabiana F. Souza ${ }^{2 *}$
}

\begin{abstract}
Moura D.S., Lourenço T.T., Moscardini M.M., Scott C., Fonseca P.O. \& Souza F.F. 2011. [Morphological aspects of ovine cervix.] Aspectos morfológicos da cérvice de ovelhas. Pesquisa Veterinária Brasileira 31(Supl.1):33-38. Hospital Veterinário, Universidade de Franca, Av. Dr. Armando Salles de Oliveira 201, Parque Universitário, Franca, SP 14404-600, Brazil. E-mail: fafesouza@unifran.br

The major barrier to accomplishment of artificial insemination in ewes is the anatomy of the cervix combined with low viability and survival of frozen ram semen. Thus, the aim of the present study was to evaluate the morphology of the cervix in ewes. Eighty-one specimens were obtained from a slaughterhouse and the following characteristics were evaluated: type of external cervical os, cervical size, integrity and interdigitation of cervical rings, gross characteristics and size of the ovaries (follicles and corpus luteum), and the time spent to pass an insemination catheter through the lumen of the cervix. The most frequent was the slit type cervical opening and grade II internal ring arrangement. The time spent to pass the insemination catheter through the cervix was 6 minutes and 15 seconds, and the dye was spread throughout the cervical lumen reaching the uterus in most sheep. The average values of cervical opening diameter and cervical length were $0.68 \mathrm{~cm}$ and $4.4 \mathrm{~cm}$ respectively. Ovarian follicular activity was found in $75 \%$ of the ewes. A positive correlation was established between some of the variables. We conclude that cervical opening size is influenced by estrogen, slit type cervical os and grade III cervical ring arrangement; also, the greater length of the cervix was associated with greater difficulty to pass the insemination catheter.
\end{abstract}

INDEX TERMS: Sheep, artificial insemination, cervical morphology.

RESUMO.- A principal barreira para a aplicação da inseminação artificial transcervical é a anatomia cervical aliada à baixa viabilidade e sobrevida do sêmen ovino congelado. Assim, este experimento teve como objetivo estudar a morfologia da cérvice de ovelhas. Para tal, foram adquiridas, em matadouro, 81 peças do trato reprodutor de ovelhas, nas quais se avaliou a morfologia cervical, segundo as seguintes características: tipo de óstio cervical, mensuração do tamanho da cérvice, integralidade e interdigitação entre os anéis das pregas cervicais, tamanho e características macroscópicas dos ovários (folículos e corpo lúteo) e tempo da passagem do aplicador de sêmen pela cérvice. Foi identificada maior frequência do tipo liso de abertura da cérvice e integralidade e interdigitação dos anéis grau II. 0 tempo de passagem do aplicador pela cérvice foi em

\footnotetext{
${ }^{1}$ Recebido em 14 de março de 2011.

Aceito para publicação em 7 de november de 2011.

${ }^{2}$ Hospital Veterinário, Universidade de Franca (Unifran), Av. Dr. Armando Sales de Oliveira 201, Parque Universitário, Franca, SP 14404-600, Brasil.*Autor para correspondência: fafesouza@unifran.br
}

média de seis minutos e 15 segundos, sendo que o corante aplicado se difundiu por todo o canal, atingindo o útero na maioria das ovelhas. A média do diâmetro da abertura cervical foi de $0,68 \mathrm{~cm}$ e o comprimento cervical de $4,4 \mathrm{~cm}$. A atividade folicular ovariana foi encontrada em $75 \%$ das fêmeas. Foi possível estabelecer várias correlações entre as variáveis. Conclui-se que o tamanho da abertura cervical sofre influência estrogênica, e o tipo liso de abertura cervical, o grau III de integralidade e interdigitação dos anéis e o maior comprimento da cérvice foram associados à maior dificuldade de passar o cateter no lúmen cervical.

TERMOS DE INDEXAÇÃO: Ovinos, inseminação-artificial, morfologia cervical.

\section{INTRODUÇÃO}

Inúmeras tentativas já foram realizadas para superar a barreira cervical em ovelhas e alcançar o útero, tendo como objetivo a inseminação artificial (IA). Isto se deve a anatomia da cérvice, a qual é o principal fator que dificulta a IA 
transcervical nesta espécie (Moses et al. 1997, Granados et al. 2006).

A cérvice das ovelhas é tubular longa, composta por tecido fibroso, predominantemente, tecido conjuntivo e pouco tecido muscular (Cruz Jr 2006). O lúmen é convoluto e tortuoso devido a presença de 4 a 7 anéis cervicais, os quais possuem as extremidades voltadas para a região caudal, servindo como uma barreira física a contaminantes externos (Dun 1955, Fukui \& Roberts 1978, Kaabi et al. 2006). 0 comprimento da cérvice da ovelha varia de 5,7 a $10 \mathrm{~cm}$ e é influenciado pela raça, idade, número de partos e estado fisiológico (Abusineina 1969, Halbert et al. 1990, Kaabi et al. 2006).

Kershaw et al. (2005) encontraram diferenças no comprimento da cérvice e profundidade de penetração com o catéter em ovelhas e borregas, durante a estação reprodutiva e o anestro estacional. Neste estudo, o comprimento médio foi de $5,35 \mathrm{~cm}$, com variação de 2,5 a $10,5 \mathrm{~cm}$, mas não foi relacionado à idade dos animais, ao ciclo reprodutivo ou ao tipo de abertura cervical. Porém, o estágio do ciclo estral afetou a penetrabilidade da cérvice, sugerindo um grau de relaxamento maior durante o estro.

0 óstio externo cervical está localizado na parte ventral da vagina e seu lúmen é fechado por proeminências e depressões recíprocas da túnica mucosa (Getty 1986). A morfologia da abertura cervical externa também difere entre as fêmeas, a qual se projeta para dentro da porção cranial da vagina, e é recoberta por uma ou mais pregas de tecido fibroso (Dun 1955). Halbert et al. (1990) descreveu 4 tipos de morfologia da abertura cervical: bico de pato (duas pregas opostas), flap (uma prega), roseta (conjunto de pregas) e espiral (tecido em formato de espiral). Posteriormente, Kershaw et al. (2005) identificou mais um tipo, o qual foi denominado liso (fenda); neste tipo não há protusão da cérvice em direção a vagina anterior sendo a abertura em formato de fenda.

O lúmen cervical é uma estrutura convoluta consistindo de seis pregas circulares. A segunda prega é excêntrica em relação às outras que são concêntricas (Moré 1984). As pregas cervicais apresentam formato de funil assimétrico (Halbert et al. 1990), com um fundo de saco em todos os anéis (Cruz Jr 2006).

Windsor (1995) demonstrou que a taxa de penetração cervical não é afetada pelo aumento do intervalo entre o parto e a IA (12-26 semanas, $n=60)$, mas quanto maior o número de partos, maior a taxa de penetração da cérvice $(n=197, p<0,05)$. Apesar disto, não foi identificada influência do tratamento hormonal (sincronização) sobre a taxa a penetração cervical $(n=101)$, mas durante a estação reprodutiva a penetração da cérvice foi facilitada.

Estudos têm demonstrado que a dificuldade e o maior tempo de manipulação cervical, no qual é empregada força excessiva, pode exercer efeito negativo sobre os resultados da transferência de embriões (Wulster-Radcliffe et al. 1999). Além disso, o estresse sofrido pelo tecido cervical pode induzir a produção de compostos espermicidas (Hawk 1983), podendo ter efeitos negativos semelhantes sobre os embriões (Wulster-Radcliffe et al. 1999).
Naqvi et al. (2003) estudaram as características morfológicas da cérvice de borregas e ovelhas adultas da região tropical semi-árida da Índia. Estes autores verificaram efeito da idade na anatomia cervical, havendo diferenças significativas no comprimento e peso da cérvice, altura e diâmetro da $1^{\underline{a}}$ e $2^{\underline{a}}$ pregas, diâmetro interno da $2^{\underline{a}}$ prega e distância entre o óstio externo da cérvice e a $1^{\mathrm{a}}$ e $2^{\mathrm{a}}$ prega. Diferenças não foram observadas entre o número de pregas e o diâmetro da $1^{\underline{a}}$ prega entre as borregas e as ovelhas adultas. A ampla variação entre as fêmeas, na anatomia cervical pode explicar os diferentes sucessos da IA por via transcervical (Kershaw et al. 2005).

Segundo Megham (2003) a morfologia cervical deve ser profundamente estudada para desenvolver equipamentos que facilitem a inseminação artificial transcervical de ovelhas e que possam transpassar a cérvice, com mínimas lesões, impedindo qualquer liberação de compostos espermicidas (Hawk 1983), que fatalmente diminuirão a fertilidade.

Considerando a importância que a criação de ovinos representa hoje no país e sabendo da dificuldade da aplicação de biotécnicas da reprodução nesta espécie objetivou-se estudar a morfologia (comprimento, profundidade, tipo e número de pregas cervicais e características do óstio externo da cérvice) da cérvice de ovelhas e correlacioná-las ao período do ciclo estral (fase luteal e não-luteal) de acordo com a morfologia dos ovários.

\section{MATERIAL E MÉTODOS}

Foram utilizadas 81 peças do sistema reprodutor feminino de ovelhas colhidas em matadouros comerciais de ovinos (Cow Pig, Boituva/SP, e Dom Pig, São Manuel/SP). Imediatamente após o abate, as peças foram colocadas em sacos plásticos e encaminhadas até o Laboratório de Reprodução Animal, onde foram congeladas e avaliadas em uma única oportunidade.

As peças foram descongeladas à temperatura ambiente, lavadas e todo o sistema reprodutor foi dissecado, retirando-se todo tecido adiposo e ligamentos. 0 sistema reprodutor foi aberto no seu sentido longitudinal até a entrada da cérvice e o óstio cervical externo foi caracterizado de acordo com Halbert et al. (1990), modificado por Kershaw et al. (2005), em 5 tipos: bico de pato (duas pregas cervicais opostas protuíndo em direção a vagina); liso (nenhuma protusão em direção a vagina); roseta (um conjunto de pregas cervicais protuíndo em direção a vagina obscurecendo o óstio cervical); papila (uma papila protui para dentro da vagina anterior e o óstio externo da cérvice encontra-se no seu ápice); e flap (uma prega do tecido cervical protui para dentro da vagina anterior e completamente ou parcialmente recobre o óstio cervical externo, criando uma aparência de flap).

Um aplicador de sêmen de ovinos (Alta Genetics, Uberaba/ MG) foi passado até o máximo da profundidade cervical, sem que força excessiva fosse realizada. 0 tempo gasto para a passagem do aplicador através da cérvice foi monitorado por um cronômetro digital. Uma palheta francesa para envase de sêmen de $0,25 \mathrm{~mL}$ foi preenchida com corante (Giemsa), colocada no aplicador de sêmen ovino e aplicada para verificar o local do depósito. Tempos superiores a 15 minutos foram computados como este valor para efeito de cálculo.

0 trato reprodutor, na altura da cérvice e corpo do útero, foi seccionado no sentido longitudinal. 0 comprimento da cérvice foi mensurado com uma régua, desde a abertura cervical externa até 
a abertura cervical interna, na altura do corpo uterino. Neste momento, as pregas cervicais foram avaliadas quanto à morfologia, segundo a integralidade e interdigitação entre os anéis e classificadas em 3 graus, de acordo com Kershaw et al. (2005): grau I (cérvice com vários anéis cervicais alinhados ao lúmen sem nenhuma interdigitação entre os anéis); grau II (cérvice apresenta uma mistura de anéis alinhados e desalinhados ao lúmen, com alguns obscurecendo o lúmen central); e grau III (cérvice apresenta predominantemente anéis cervicais desalinhados interdigitalizando-se).

Após a dissecação dos ovários, a superfície ovariana foi avaliada macroscopicamente para a presença de folículos e corpo lúteo. Então os ovários foram cortados na sua porção média no sentido longitudinal e avaliados novamente. 0 conjunto de ovários de cada fêmea foi classificado em fase luteal (presença de corpo lúteo num dos ovários) e não-luteal (ausência de corpo lúteo nos ovários).

0 teste de correlação de Spearman, para dados não-paramétricos, foi utilizado para análise das variáveis, pois as variáveis não passaram no teste de normalidade de Kolmogorov-Smirnov. 0 nível de significância considerado foi de $\mathrm{p}<0,05$. Para tal utilizou-se o programa Sigma Stat for Windows, versão 3.0.1, 2003.

\section{RESULTADOS}

Os resultados referentes às características da cérvice de 81 peças do sistema genital de ovelhas estão representados na Quadro 1. As ovelhas foram, na maioria, da raça Santa Inês, adultas, porém os dados específicos de cada peça (idade, raça, status reprodutivo, número de partos e fase do ciclo estral) não foram fornecidos pelo matadouro.

Quanto ao tipo de abertura cervical foram identificados todos os descritos (Quadro 1), porém o mais freqüente foi o tipo liso ( $\mathrm{n}=31 ; 38,3 \%)$, o qual foi, aproximadamente, o dobro dos demais: bico de pato $(n=12,14,8 \%)$, roseta $(n=14$; $17,3 \%)$, papila $(n=11 ; 13,6 \%)$, flap $(n=13 ; 16 \%)$.

0 número de peças que apresentavam o grau II de integralidade e interdigitação entre os anéis das pregas cervicais foi maior ( $n=32,39,5 \%)$ do que o grau I $(n=28,34,6 \%)$ e III ( $n=21,25,9 \%)$. 0 grau de integralidade e interdigitação entre os anéis das pregas cervicais foi correlacionado, positivamente, ao tipo de abertura externa da cérvice $(\mathrm{r}=0,25$; $\mathrm{p}=0,026)$ e ao tempo gasto para a passagem do aplicador de sêmen $(r=0,50 ; p<0,01)$, sendo maior tempo de passagem do aplicador, nas peças com grau I e abertura cervical do tipo liso. 0 Quadro 2 apresenta a porcentagem de cada

Quadro 1. Resultados das características da cérvice de peças do sistema genital de 81 ovelhas

\begin{tabular}{|c|c|c|c|c|}
\hline Característica & Média & $\begin{array}{l}\text { Desvio } \\
\text { padrão }\end{array}$ & Máximo & Mínimo \\
\hline $\begin{array}{l}\text { Tamanho da abertura cervi- } \\
\text { cal }(\mathrm{cm})\end{array}$ & 0,68 & 0,26 & 1,40 & 0,30 \\
\hline Comprimento cervical (cm) & 4,40 & 1,73 & 11,10 & 1,00 \\
\hline $\begin{array}{l}\text { Tempo para passagem } \\
\text { pipeta* }\end{array}$ & $6^{\prime}$ e $15^{\prime \prime}$ & $6^{\prime}$ e 8" & $15^{\prime}$ & $2 "$ \\
\hline $\begin{array}{l}\text { Comprimento do ovário } \\
\text { direito }(\mathrm{cm})\end{array}$ & 1,21 & 0,41 & 2,20 & 0,40 \\
\hline $\begin{array}{l}\text { Raio do folículo no ovário } \\
\text { direito }(\mathrm{cm})\end{array}$ & 0,42 & 0,24 & 1,50 & 0,20 \\
\hline $\begin{array}{l}\text { Comprimento do ovário } \\
\text { esquerdo }(\mathrm{cm})\end{array}$ & 1,20 & 0,35 & 2,30 & 0,50 \\
\hline Raio do folículo no ovário & 0,40 & 0,20 & 1,00 & 0,10 \\
\hline
\end{tabular}

esquerdo (cm)

*(') Minutos; (") segundos.

Quadro 2. Porcentagem do grau de integralidade e interdigitação dos anéis cervicais nos diferentes tipos de abertura caudal da cérvice de 81 ovelhas adultas

\begin{tabular}{llll}
\hline Tipo de abertura cervical & Grau I & Grau II & Grau III \\
\hline Lisa (\%) & 22,6 & 38,7 & 38,7 \\
Roseta (\%) & 28,6 & 57,1 & 14,3 \\
"Flap" (\%) & 53,8 & 15,4 & 30,8 \\
Papila (\%) & 36,4 & 63,6 & 0,0 \\
Bico de pato (\%) & 50,0 & 25,0 & 25,0
\end{tabular}

grau de integralidade e interdigitação em relação ao tipo de abertura cervical.

A média do tempo para a passagem do aplicador seminal foi de seis minutos e 15 segundos, o mínimo de 2 segundos e o máximo de 15 minutos, considerando que a partir dos 15 minutos o tempo não foi anotado. 0 corante introduzido com o aplicador de sêmen se difundiu por toda a cérvice (Quadro 2), atingindo o útero, na maioria das ovelhas. Embora este dado não tenha sido anotado para todas as peças e tão pouco foi analisado estatisticamente, foi observada maior difusão do corante na cérvice com grau I de integralidade e interdigitação das pregas cervicais e menor difusão naquelas do grau III (Fig.2).

A média do diâmetro da abertura cervical externa foi $0,68 \mathrm{~cm}$, sendo os valores mínimos e máximos de $0,3 \mathrm{~cm}$ e $1,4 \mathrm{~cm}$, respectivamente. 0 comprimento médio da cérvice foi de $4,40 \mathrm{~cm}$, sendo as medidas mínimas e máximas de um a $11,10 \mathrm{~cm}$.

Quanto à atividade ovariana, aproximadamente $75 \%$ $(\mathrm{n}=61)$ das ovelhas apresentavam apenas folículos nos ovários, contra $25 \%(n=20)$ que apresentavam corpo lúteo e folículos.

Também foi observada uma correlação positiva entre o diâmetro da abertura cervical e a presença de folículos ovarianos $(r=0,30, p<0,01)$ e uma correlação negativa entre a presença de folículos e o tempo para a passagem do cateter de inseminação ( $r=-0,31, p<0,01)$.

0 tamanho dos ovários foi correlacionado, positivamente, ao tamanho da abertura (ovário direito $\mathrm{r}=0,22, \mathrm{p}=0,05 \mathrm{e}$ ovário esquerdo $r=0,27, p=0,02$ ) e comprimento da cérvice (ovário direito $\mathrm{r}=0,46, \mathrm{p}<0,01$ e ovário esquerdo $\mathrm{r}=0,58$, $\mathrm{p}<0,01)$. Também foi encontrada uma correlação positiva entre o diâmetro do maior folículo com o tempo gasto para a passagem do cateter pela cérvice $(r=0,35, p<0,01)$ e $o$ comprimento cervical $(r=0,25, p=0,03)$.

\section{DISCUSSÃO E CONCLUSÃO}

A inseminação transcervical em ovelhas não é muito comum pela complexa anatomia da cérvice ovina que diminui a taxa de penetração em $50 \%$, em função da variabilidade individual e habilidade do inseminador (Verberckmoes et al. 2001). No presente estudo, o tempo gasto para a passagem do cateter de inseminação foi superior a 10 minutos em aproximadamente $31 \%$ das ovelhas, o que dificulta a utilização destes animais para os programas de reprodução assistida, confirmando as dificuldades relatadas na literatura.

As peças utilizadas no estudo foram adquiridas em abatedouros comerciais e nenhum dado a respeito da idade, 

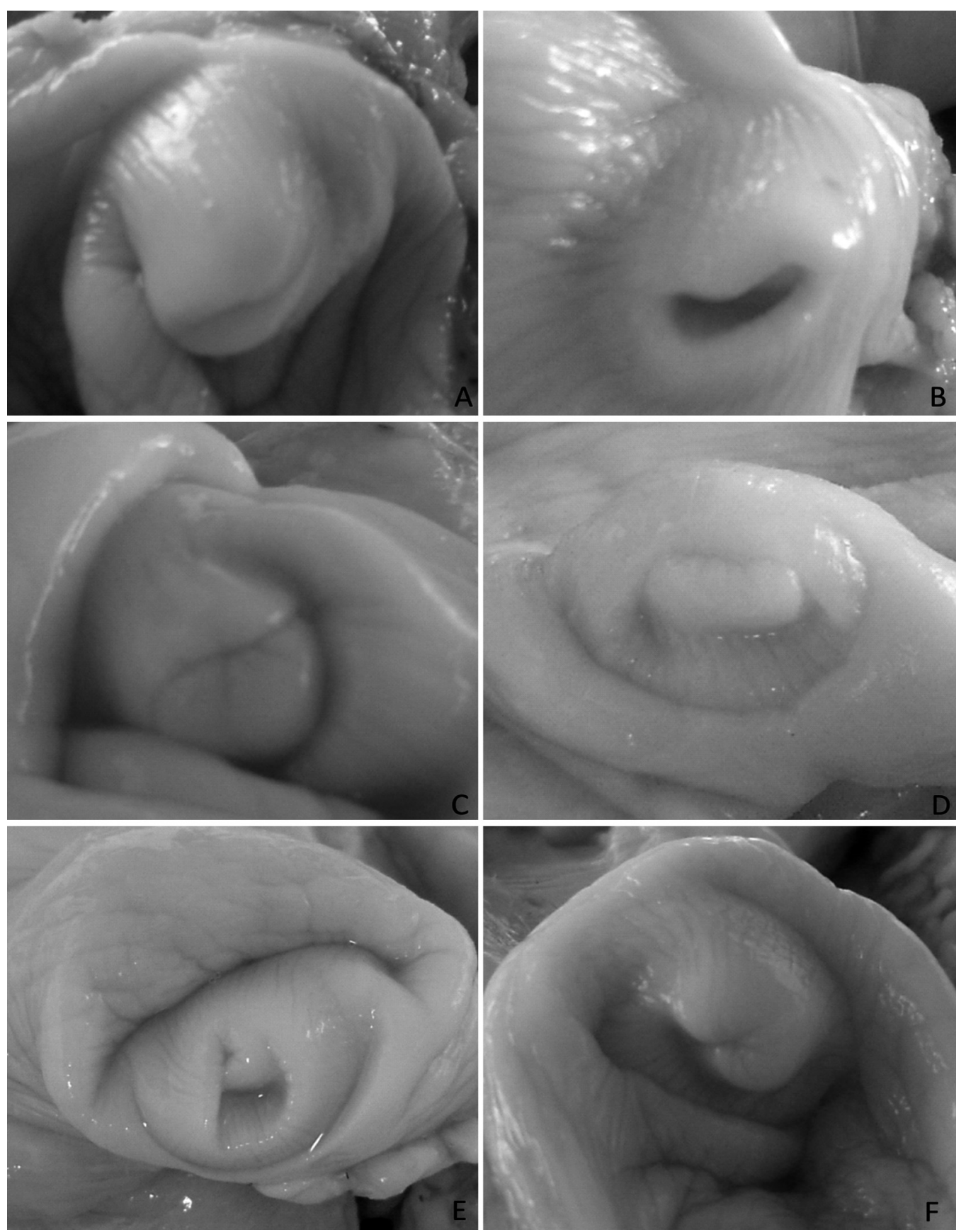

Fig.1. Morfologia da abertura cervical de ovelhas, segundo classificação proposta por Halbert et al. (1990) e modificada por Kershaw et al. (2005). (A,D) Bico de pato, (B) Lisa, (C) Roseta, (E) Papila, (F) "Flap".

raça ou status fisiológico e reprodutivo foi fornecido, o que não permitiu a correlação destes dados com os avaliados.

o comprimento cervical médio e a sua variação apresentaram-se semelhantes aos estudos de Kershaw et al. (2005) os quais encontraram comprimento médio da cérvice de $5,4 \mathrm{~cm}$, com uma variação de 2,5 a $10,5 \mathrm{~cm}$. Contudo, o valor mínimo foi inferior $(1,0 \mathrm{~cm})$ aos estudos destes autores. Já Breda et al. (2007) obteve uma maior média do comprimento cervical de $7,2 \mathrm{~cm}$ em animais com 2 a 8 anos de idade e média de 2,0 a 2,2 partos, nas raças Hampshire Down e em ovelhas mestiças Hampshire Down - Ilê de France. Da mesma forma que estes autores, o presente es- tudo encontrou uma maior porcentagem de ovelhas com grau II (aproximadamente 39,5\%) de integralidade e interdigitação dos anéis cervicais, porém esta diferença não foi tão marcante quanto às relatadas por aqueles estudos.

A média do diâmetro da abertura cervical externa $(0,68$ $\mathrm{cm}$ ) foi superior aos achados de Gultiken et al. (2009), os quais encontraram uma média de $0,28 \mathrm{~cm}$ e 0,31 em borregas e ovelhas adultas da raça Karayaka, respectivamente.

Na raça Santa Inês, Cruz Jr (2006) identificou a predominância do tipo de abertura cervical bico de pato $(69,12 \%)$, contrariando os achados aqui apresentados, nos quais foi identificado, com maior freqüência, o tipo liso de 


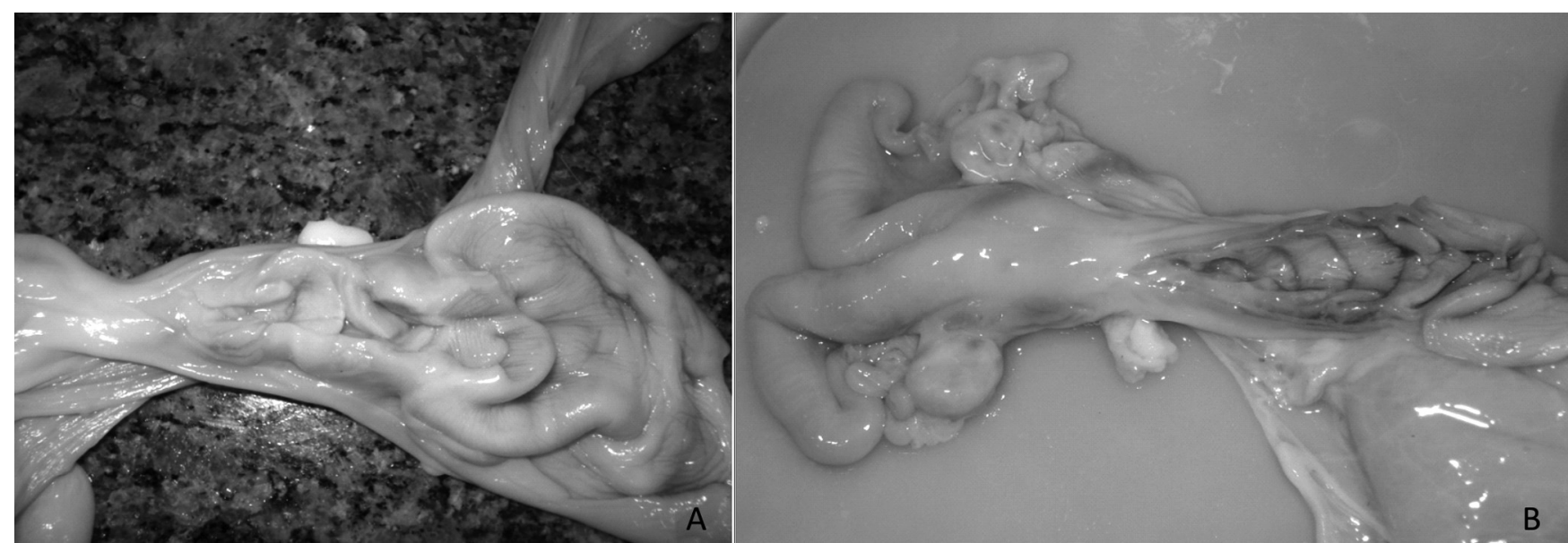

Fig.2. Penetração do corante após passagem do aplicador seminal na cérvice de ovelhas. (A) Nota-se o corante apenas nas primeiras pregas cervicais; tal cérvice foi classificada como de grau III, segundo a integralidade e interdigitação das pregas cervicais. (B) Nota-se o corante por toda a cérvice, a qual foi classificada como de grau I, segundo a integralidade e interdigitação das pregas cervicais.

abertura cervical. Na raça Corriedale o tipo de abertura de cérvice mais observado foi "flap". Nas raças Suffolk, Cheviot e Dorset houveram maior freqüência do tipo roseta (Cruz Jr 2006). Também Souza (1993) verificou maior freqüência da abertura do tipo flap em ovelhas Corriedale $(69,12 \%)$ e Ideal e Corriedale $(52,21 \%)$ em relação aos demais tipos. Diferente dos resultados de outros estudos (Kershaw et al. 2005, Breda et al. 2007), foi possível verificar uma correlação positiva entre o grau de integralidade e interdigitação dos anéis ao tipo de abertura cervical. Foi gasto maior tempo para a passagem do aplicador de sêmen nas ovelhas com maior grau de integralidade e interdigitação dos anéis cervicais, o que comprova a necessidade da seleção de animais para a aplicação da IA transcervical, haja visto que esta característica não se altera em função de nenhum parâmetro.

0 principal achado deste estudo foi à correlação entre o grau de integralidade e interdigitação entre os anéis das pregas cervicais, o tipo de abertura e o tempo gasto para a passagem do aplicador de sêmen. Foi identificado maior grau de dificuldade na passagem do cateter cervical, nas peças que apresentavam o tipo de abertura lisa, sendo as mesmas com maior grau de integralidade e interdigitação dos anéis cervicais (grau I). Nestas fêmeas foi encontrado o dobro do grau II e III em relação ao grau I. Nas ovelhas com tipo de abertura roseta, o grau II foi predominante. Nos tipos "flap" e bico de pato o grau I foi aproximadamente o dobro dos outros graus. E no tipo papila, o grau II foi quase o dobro do grau I, porém nenhum grau III. Numa escala de penetrabilidade, descrito pelo tempo gasto para a passagem do cateter pela cérvice, o tipo liso é o de menor penetrabilidade, sendo seguido pelos tipos roseta, "flap", papila e bico de pato com maior penetrabilidade. Embora não existam relatos da correlação entre a penetrabilidade e o tipo de abertura da cérvice (Naqvi et al. 2003), e nem há informações a respeito da raça, idade e status fisiológico dos animais estudados, sugere-se que estas ovelhas com abertura cervical do tipo liso, possam ser fêmeas contra-indicadas a IA por via transcervical.

Windsor (1995) correlacionou a taxa de penetrabilidade ao número de partos, estro e a estação do ano e re- velou que quanto maior o número de partos, maior a taxa de penetração da cérvice. Porém, não identificou nenhuma influência hormonal na penetrabilidade cervical. Contrariamente, Donavan et al. (2004) afirma que durante a estação reprodutiva a penetração é facilitada. No estudo aqui apresentado, embora as peças tenham sido colhidas em diferentes épocas, não foi possível correlacionar os achados à estação reprodutiva, devido ao pequeno número de peças, distribuídas em cada período do ano.

Kershaw et al. (2005) identificou maior penetrabilidade da cérvice, em ovelhas na fase não-luteal. Contrariamente, neste estudo, a penetrabilidade cervical foi avaliada pelo tempo gasto em introduzir o cateter de inseminação artificial, sendo assim, a penetrabilidade foi menor em fêmeas com folículos ovarianos maiores.

Nas condições do estudo, conclui-se que o tamanho da abertura cervical é maior quando o ovário apresenta folículos em desenvolvimento. Além disso, a dificuldade em passar a cérvice com o aplicador de sêmen é maior quando existe maior grau de integralidade e interdigitação entre os anéis das pregas cervicais, nas fêmeas que apresentam maior comprimento da cérvice e naquelas com a abertura cervical do tipo liso.

Agradecimentos.- Aos Frigoríficos Cow Pig (Boituva/SP) e Dom Pig (São Manuel/SP) e a FAPESP (Fundação de Amparo a Pesquisa do Estado de São Paulo) pela concessão da bolsa de estudos (Proc.2007/05147-9 e 2009/01102-6).

\section{REFERÊNCIAS}

Abusineina M.E. 1969. Effect of pregnancy on the dimensions and weight of the cervix uteri of sheep. Brit. Vet. J. 125:21-24.

Breda J.C., Giacomeli A.M., Kozicki L.E., Sotomaior. C., Milczewski V., Caldas J.S., Schwartz M.G., Carli L. \& Zat R. 2007. Dados de alguns parâmetros anatômicos do aparelho reprodutivo de ovelhos da raça Hampshire down e mestiças hampshire down e mestiças hampshire down-ile de France. Rev. Acad. 5:237-242.

Cruz Jr C.A. 2006. Caracterização anatômica e histológica da cérvix de ovelhas da ração Santa Inês. Dissertação de Mestrado em Ciências Agrárias, Faculdade de Agronomia e Medicina Veterinária, Universidade de Brasília, Brasília, DF. 45p. 
Donavan A., Hanrahan J.P., Kummen E., Duffy P. \& Boland M.P. 2004. Fertility in the ewe following cervical insemination with fresh or frozen-thawed semen at a natural or synchronized oestrus. Anim. Reprod. Sci. 84:359-368.

Dun R. 1955. The cervix of the ewe. Its importance in artificial insemination of sheep. Aust. Vet. J. 31:101-103.

Fukui Y. \& Roberts E. 1978. Further studies on non-surgical intrauterine technique for artificial insemination in the ewe. Theriogenology 10:381-393.

Getty R. 1986. Anatomia dos Animais Domésticos. 5a ed. Guanabara, São Paulo, p.894-895.

Granados L.B.C., Dias A.J.B. \& Sales M.P. 2006. Aspectos gerais da reprodução de caprinos e ovinos. Projeto PROEX/UENF, Campos dos Goytacazes, RJ.

Gultiken N., Gultiken M.E., Anadol E., Kabak M. \& Findik M. 2009. Morphometric study of the cervical canl in Karayaka ewe. J. Anim. Vet. Adv. 8:2247-2250.

Halbert G.W., Dobson H., Walton J.S. \& Buckrell B.C. 1990. The structure of the cervical canal of the ewe. Theriogenology 33:977-992.

Kaabi M., Alvarez A., Anel E., Chamorro C.A., Boixo J.C., Paz P. \& Anel L. 2006. Influence of breed and age on morphometry and deepth of inseminating catheter penetration in the ewe cervix: a postmortem study. Theriogenology 66:1876-1883.

Kershaw M.C., Khalid M., Mcgowan M.R., Ingram K., Leethongdee S., Wax G. \& Scaramuzzi R.J. 2005. The anatomy of the sheep cervix and its influence on the transcervical passage of an inseminating pipette into the uterine lumen. Theriogenology 64:1225-1235.
Hawk H.W. 1983. Sperm survival and transport in the female reproductive tract. J. Dairy Sci. 66:2645-2660.

Megham C., Radcliffe W., Wang S. \& Lewis G. S. 2003. Transcervical artificial insemination in sheep effects of a new transcervical artificial insemination instrument and traversing the cervix on pregnancy and lambing rates. Theriogenology 24:991-1002.

Moré J. 1984. Anatomy and histology of the cervix uteri of the ewe: new insights. Acta Anatom. 120:156-159.

Moses D., Martínez A.G., Iorio G., Valcárcel A., Ham A., Pessi H., Castanón R., Maciá A. \& Heras M.A. 1997. A large-scale program in laparoscopic intrauterine insemination with frozen-thawed sêmen in Australian Merino sheep in Argentine Patagonia. Theriogenology 48:651-657.

Naqvi S.M.K., Pandey G.K., Gautam K.K., Joshi A., Geethalakshmi V. \& Mittal J.P. 2003. Evaluation of gross anatomical features of cervix of tropical sheep using cervical silicone moulds. Anim. Reprod. Sci. 85:337344.

Souza M.I.L. 1993. A via cervical na inseminação artificial ovina com sêmen congelado. Dissertação de Mestrado em Medicina Veterinária, Universidade Federal de Santa Maria, RS. 47p.

Verberckmoes S., De Pauw I., Van Soon A., Vanroose G., Laevens H. \& Kruif A. 2001. Cervical insemination in sheep. Theriogenology 70:475-480.

Windsor D.P. 1995. Factors influencing the success of transcervical insemination in Merino ewes. Theriogenology 43:1009-1018.

Wulster-Radcliffe M.C., Costine B.A. \& Levis G.S. 1999. Estradiol-17ßoxytocin-induced cervical dilation in sheep: Application to transcervical embryo transfer. J. Anim. Sci. 77:2587-2593. 